\title{
Akupunkturleistungen korrekt abrechnen
}

- Die Leistungsbeschreibung der Nr. 30791 EBM sieht die Durchführung einer Körperakupunktur und ggf. Revision des Therapieplans gemäß den Qualitätssicherungsvereinbarungen nach $\S 135$ Absatz 2 SGB V zur Behandlung bei den Indikationen chronische Schmerzen der Lendenwirbelsäule oder chronische Schmerzen eines oder beider Kniegelenke durch Gonarthrose vor. Die Leistung kann je dokumentierter Indikation bis zu zehnmal, mit besonderer Begründung bis zu 15-mal im Krankheitsfall berechnet werden.

Dies bedeutet, dass die Nr. 30791 EBM im entsprechenden Einzelfall bei Vorliegen beider Indikationen bis zu 30-mal mit Begründung innerhalb eines Krankheitsfalls berechnet werden kann. Wichtig ist deshalb, dass die entsprechenden Diagnoseschlüssel nach ICD $10 \mathrm{GM}$ angegeben werden. Ab der 11. Sitzung muss je Indikation eine Begründung für die Abrechnung hinzugefügt werden. Gemäß den Richtlinien zur Qualitätssicherung Akupunktur sind die je Indikation bezogenen zehn Behandlungen innerhalb von sechs Wochen durchzuführen. Verlängert sich die Be-

\section{Tabelle}

EBM Legende (Kurzform)

Punkte

30791 Durchführung einer Körperakupunktur und ggf. Revision des Therapieplans bei chronischen Schmerzen der Lendenwirbelsäule oder eines oder beider Kniegelenke durch Gonarthrose, je dokumentierter Indikation bis zu zehnmal, mit besonderer Begründung bis zu 15-mal im Krankheitsfall.

Eignung der Prüfzeiten: Tagesprofil 10 Minuten.

handlung aus besonderen Gründen auf 15 Sitzungen, sind diese in maximal zwölf Wochen abzuschließen.

\section{MMW Kommentar}

Beachtenswert bei der Berechnung der Leistung ist auch der Begriff des "Krankheitsfalls". Danach ist in der EBM-Abrechnung die Gesamtdauer einer von derselben Arztpraxis (Vertragsarzt, Vertragspsychotherapeut, Berufsausübungsgemeinschaft, Medizinisches Versorgungszentrum) an demselben Versicherten ambulant zu Lasten derselben Krankenkasse vorgenommene Behandlung zu verstehen, die sich über mehr als drei und bis zu vier Quartale erstreckt. Frühestens mit Be- ginn des vierten Quartals, das auf das Kalendervierteljahr folgt, in dem der Krankheitsfall eingetreten ist, kann von einem neuen Krankheitsfall ausgegangen werden, wenn eine weitere oder erneute Behandlung erforderlich ist. Wurde z. B. bei einem Patienten im Laufe des 2. Quartals 2012 eine solche Akupunkturbehandlung durchgeführt, endet der Krankheitsfall nach der Definition im Bundesmantelvertrag (BMV/AEV) am 31. März 2013. Bezogen auf dieselbe Erkrankung kann somit frühestens am ersten Tag des darauffolgenden 2. Quartals 2013 abrechnungstechnisch ein neuer Krankheitsfall und damit eine erneute Akupunkturbehandlung beginnen.

\section{KBV: Arzneimittelsoftware hilft mit neuen Funktionen}

_ Ab dem 3. Quartal 2012 ändert sich die Software, die in den Praxen zum Verordnen von Arzneimitteln eingesetzt werden kann. Hintergrund hierfür sind neue Softwarevorgaben, auf die sich die KBV und der Spitzenverband Bund der Krankenkassen (SpiK) verständigt haben.

Mit der neuen Software stehen mehr Informationen in elektronischer Form zur Verfügung. So weist die neue Software auch auf Verordnungseinschränkungen und Verordnungsausschlüsse hin, die aus der Arzneimittel-Richtlinie bei konkreten Präparaten resultieren. Da der KBV vom Gesetzgeber die Aufgabe übertragen wurde, die jeweilige Arzneiverordnungssoftware eines Anbieters zu zertifizieren, müssen diese $a b$
1. Juli 2012 die Möglichkeit einräumen, auch werbefreie Versionen zu nutzen. Diese dürften in der Regel allerdings etwas teurer ausfallen, da die Einnahmen aus der Werbung für das Softwareunternehmen wegfallen.

\section{MMW Kommentar}

Ist die Verordnung eines Präparates nur eingeschränkt möglich, weist die neue Software darauf hin. Man kann dann die vollständigen Texte aus der Arzneimittel-Richtlinie oder Hinweise aus Arzneimittelvereinbarungen anzeigen lassen. Das neue SoftwareSystem bietet zudem die Möglichkeit, diese Informationen abzuschalten, z. B. wenn man sie schon kennt. Sie bleiben jedoch im Sys- tem hinterlegt, sodass man bei Bedarf nachlesen kann. Auch weist die Software jetzt nur noch bei Erwachsenen und bei Kindern ab zwölf Jahren darauf hin, dass es sich um ein OTC-Präparat handelt und damit eine Verordnung zu Lasten der gesetzlichen Krankenversicherung ausscheidet. Ferner enthält die neue Software Informationen zu Rabattverträgen. So wird z. B. sichtbar, ob ein rabattiertes Arzneimittel, das zur Substitution geeignet ist, existiert.

Die wichtigste Information zu diesem Thema fehlt aber auch bei der neuen Software: Die Preise rabattierter Medikamente und die Höhe des Rabattes bleiben das Geheimnis der Krankenkasse und des jeweiligen pharmazeutischen Unternehmen. 